\title{
Increased expression of Ectodysplasin A2 Receptor EDA2R is the most remarkable and ubiquitous aging-related transcriptional hallmark
}

\section{Maria Chiara Barbera}

Mario Negri Institute for Pharmacological Research

\section{Laura Di Rito}

Mario Negri Institute for Pharmacological Research

\section{Luca Guarrera}

Mario Negri Institute for Pharmacological Research https://orcid.org/0000-0001-7475-3345

\section{llaria Craparotta}

Mario Negri Institute for Pharmacological Research

\section{Arianna Vallerga}

INSTITUTE OF ONCOLOGY RESEARCH (IOR)

\section{Margherita Romeo}

Institute of Clinical Chemistry and Laboratory Diagnostic Medical Faculty, Heinrich Heine University and the IUF-Leibniz Research Institute for Environmental Medicine https://orcid.org/0000-0002-3020-8545

\section{Sarah Mapelli}

Humanitas Clinical and Research Center-IRCCS https://orcid.org/0000-0002-0129-2055

Marco Bolis ( $\sim$ marco.bolis@ior.usi.ch )

INSTITUTE OF ONCOLOGY RESEARCH (IOR) https://orcid.org/0000-0003-4377-5079

\section{Brief Communication}

Keywords:

Posted Date: August 4th, 2021

DOI: https://doi.org/10.21203/rs.3.rs-774469/v1

License: (c) (i) This work is licensed under a Creative Commons Attribution 4.0 International License.

Read Full License 
1 ARTICLE TYPE: Brief Communication

2

3 TITLE: Increased expression of Ectodysplasin A2 Receptor EDA2R is the most

4 remarkable and ubiquitous aging-related transcriptional hallmark

5

6 TOTAL WORD COUNT: 1550

7 ABSTRACT: 70

8 MAIN TEXT: 549

9 FIGURE LEGENDS: 538

10 REFERENCES: 393

11

12

\section{Graphical Abstract}
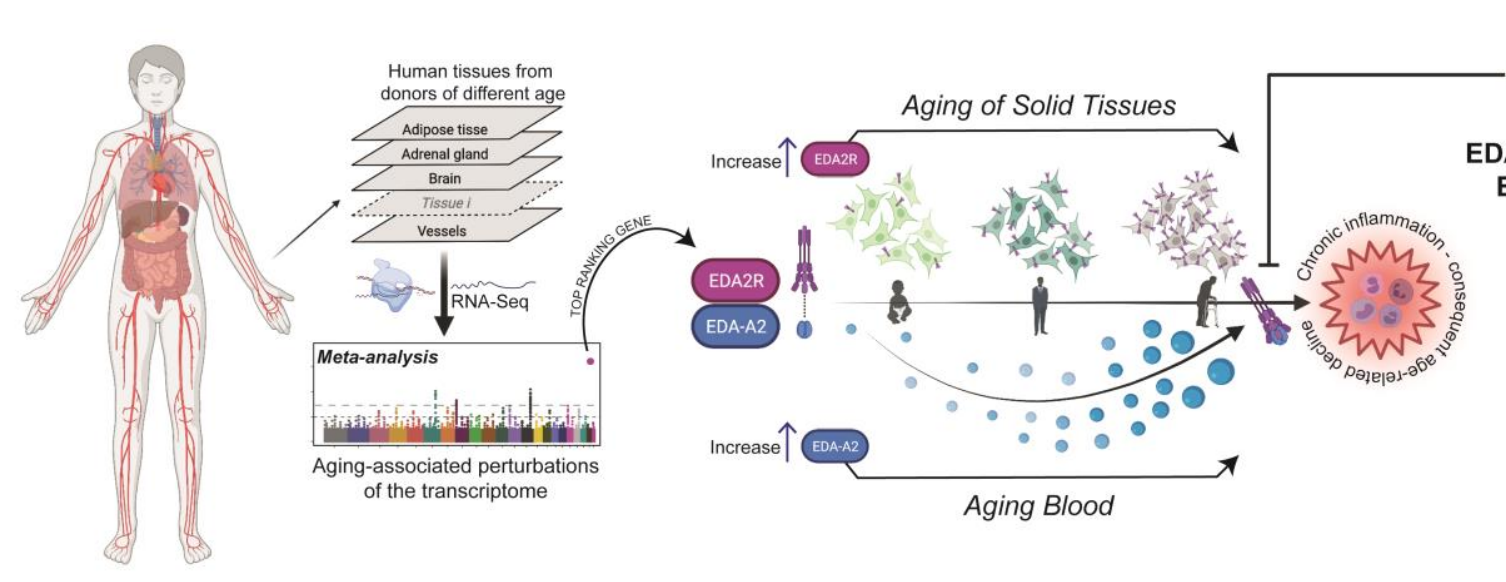
17 TITLE: Increased expression of Ectodysplasin A2 Receptor EDA2R is the most

18 remarkable and ubiquitous aging-related transcriptional hallmark

19

20 M. Chiara Barbera ${ }^{1^{*}}$, Laura Di Rito ${ }^{1^{*}}$, Luca Guarrera ${ }^{1^{*}}$, Ilaria Craparotta ${ }^{1}$, Arianna

21 Vallerga ${ }^{2,3,4}$, Romeo M. ${ }^{6}$, Sarah N. Mapelli ${ }^{5}$, Marco Bolis ${ }^{1,2,3,4, @ ~}$

22

23 * These authors contributed equally to this work

$24 @$ Correspondence to: marco.bolis@ior.usi.ch, marco.bolis@ marionegri.it

$26{ }^{1}$ Computational Oncology Unit, Department of Oncology, 'Mario Negri' Institute for Pharmacological

27 Research IRCCS, Via Mario Negri 2, 20156 Milano, Italy

28 Institute of Oncology Research, Faculty of Biomedical Sciences, USI, Bellinzona, TI 6500,

29 Switzerland

30 3Università Della Svizzera Italiana (USI), Faculty of Biomedical Sciences, Lugano $\mathrm{CH}-1011$,

31 Switzerland.

$32{ }^{4}$ Swiss Institute of Bioinformatics, Bioinformatics Core Unit, Bellinzona, TI 6500, Switzerland

$33{ }^{5}$ Humanitas Clinical and Research Center-IRCCS, Milan, Italy.

34 Institute of Clinical Chemistry and Laboratory Diagnostic Medical Faculty, Heinrich Heine

35 University and the IUF-Leibniz Research Institute for Environmental Medicine, Düsseldorf, 40225

36 Germany 
44 ABSTRACT

45 We report that increased mRNA-expression of Ectodysplasin-A2-Receptor (EDA2R)

46 is the most remarkable aging-associated transcriptional perturbation in humans,

47 and that its induction is confirmed in murine-models of Hutchinson-Gilford progeria

48 syndrome and in other species. Up-regulation of EDA2R occurs in all solid-tissues

49 and is complemented by the progressive transcriptional increase of its ligand in

50 bloodstream. Strengthening of EDA2R/EDA-A2 signaling may exacerbate

51 inflammation in ageing individuals which might be prevented by EDA2R/EDA-A2

52 blockade.

54 MAIN TEXT

55 Ectodysplasin A2 Receptor is part of the tumor necrosis factor receptor (TNFR)

56 superfamily which selectively binds to $E D A-A 2$, a protein encoded by an alternative

57 splicing isoform of EDA (Ectodysplasin A) gene. EDA2R receptor has been cataloged as

58 a target of $T P 53^{1,2}$ and EDA2R/EDA-A2 signaling has been observed to mediate activation

59 of JNK, NF-kB pathways ${ }^{3,4}$ and to promote inflammation, apoptosis and cell death ${ }^{2,5-9}$.

60 Indeed, in diabetic kidneys, EDA2R has been shown to mediate podocyte injury through

61 ROS generation ${ }^{10}$. Additionally, several studies indicated that the gene locus of EDA2R is

62 associated with age-associated androgenetic alopecia (AGA), which is a known common

63 heritable polygenic disorder ${ }^{11,12}$. Moreover, EDA2R mRNA expression was reported to be

64 elevated in the aging lungs ${ }^{13}$.

65 To identify genes whose expression could be positively correlated with the increasing

66 age of individuals, our group selectively screened the Genotype-Tissue Expression

67 database (GTEx) ${ }^{14}$ looking for transcriptional changes occurring in a tissue-independent

68 fashion. To this purpose, we went through a rigorous multi-step bioinformatics analysis

69 procedure which aimed at minimizing possible confounding factors such as gender 
70 differences or the precise anatomical sub-localization of the sample of origin (See

71 Methods section). EDA2R emerged as the most noticeable outlier of the analysis, as its

72 expression resulted to be strikingly well correlated to the increasing age of donors in a

73 tissue-type independent manner. Indeed, EDA2R appeared among the very top correlated

74 genes in all solid tissues evaluated (Fig1A, Supp.Fig1A, Supp.Table1) and its median

75 correlation coefficient ranked first in the integrated pan-tissue analysis (Fig1B).

76 Furthermore, strong induction of Eda2r also emerged in aging tissues of rats and mice,

77 suggesting a non-species-specific behavior (Fig1A-B, Supp.Fig1B, Supp.Table2). Next,

78 to further strengthen our findings, we investigated transcriptional changes occurring in

79 murine models of Hutchinson-Gilford progeria syndrome (HGPS), a recognized proxy of

80 accelerated aging in humans, to determine if Eda2r induction could be peculiar to the

81 disease. Remarkably, the analysis revealed Eda2ritself as one of the very top genes being

82 most significantly up-regulated in the aorta of HGPS mice compared to the age matched

83 wild-type counterpart (2 months old), confirming thereby a deep connection to the aging

84 processes (Fig2A-B, Supp.Table3). Altogether, our findings strongly support a unique

85 tissue-independent association between aging and EDA2R mRNA levels.

86 Conversely, transcription from the EDA locus, which codes for the EDA2R ligand, did

87 not correlate substantially with age in most solid tissues, however, age-mediated induction

88 of EDA turned evident in blood-derived samples (Fig3A), showing a different behavior

89 compared to EDA2R receptor. In particular, the EDA-A2 isoform resulted to be more

90 expressed in venous blood compared to the EDA-A1 counterpart (Supp.Fig1C,

91 Supp.Table1), being the former the selective ligand of EDA2R. As increased EDA-

92 A2/EDA2R mediated signaling could trigger inflammatory responses, we questioned

93 whether baseline activity of inflammatory pathways would also correlate to age in a tissue-

94 independent manner. Our observations confirmed this last hypothesis in most tissues

95 (Fig3B), indicating inflammation as one of the top-ranking biological process associated 
96 with aging, suggesting thereby a possible link with EDA2R activity.

97 Taken together, our results disclose a single-gene biomarker, impressively associated

98 to increasing age in every individual tissue, solving a scientific inquiry which has been

99 previously answered exclusively with DNA methylation models ${ }^{15,16}$. More importantly, the

100 EDA2R/EDA-A2 axis, with its ligand-receptor activity and downstream signaling pathways,

101 suggest novel targets for the development of compounds aimed at slowing down chronic

102 inflammation and consequent age-associated decline.

103

104 Author Contributions

105 M.B., C.B., and S.M. originally developed the concept and further elaborated on it. M.B.,

106 L.D.R., and L.G. carried out all bioinformatic analyses. M.B., S.M., I.C., M.R., wrote the

107 manuscript and designed all figures.

108

109 Declaration of Interests

110 The authors declare no conflicts of interest.

\section{Correspondence}

113 Correspondence and requests should be addressed to M.B., (marco.bolis@ior.usi.ch ;

114 marco.bolis@marionegri.it )

115

116

117

118 


\section{Figure 1}

a

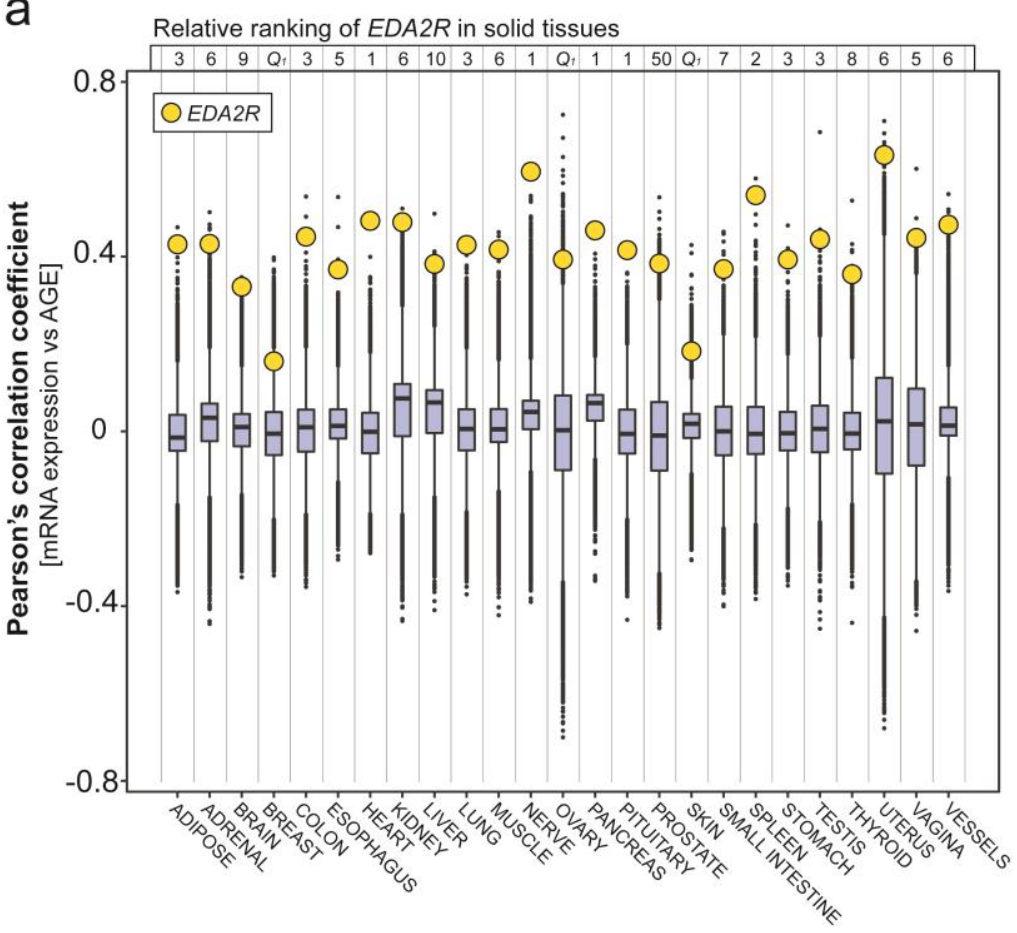

b

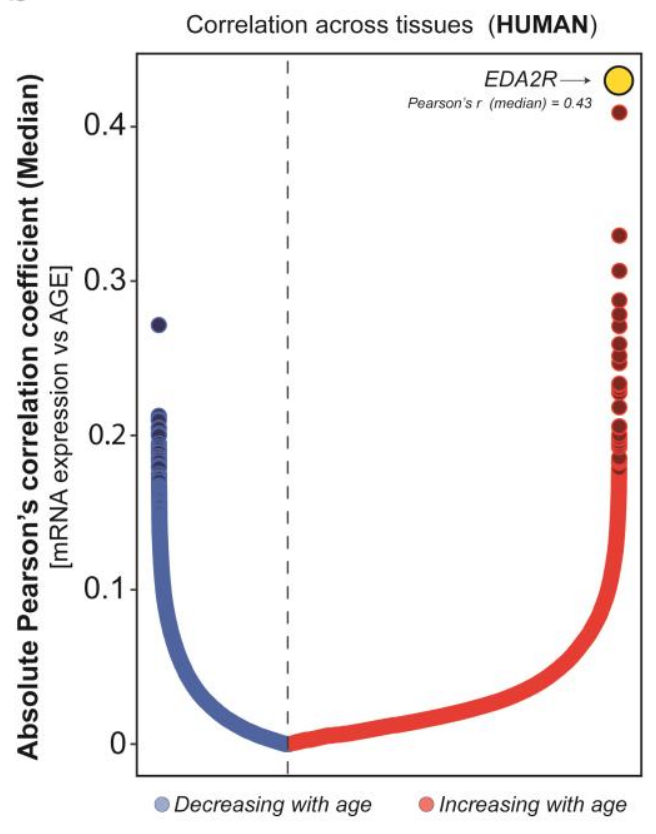

C

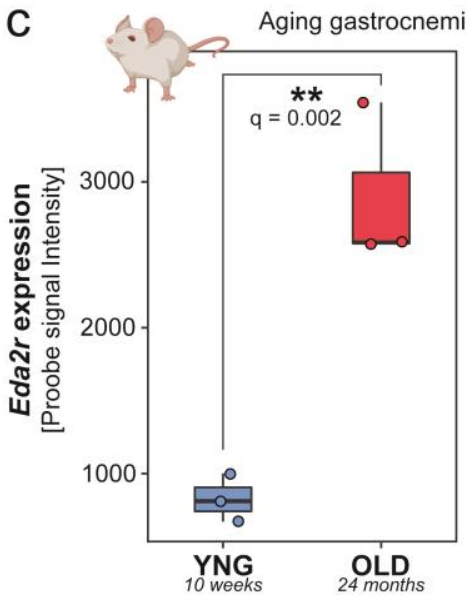

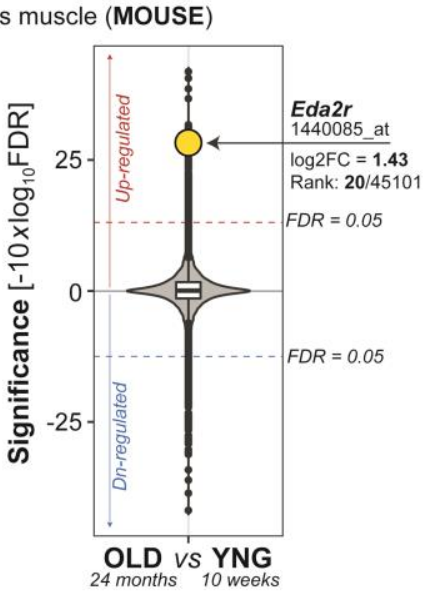

d

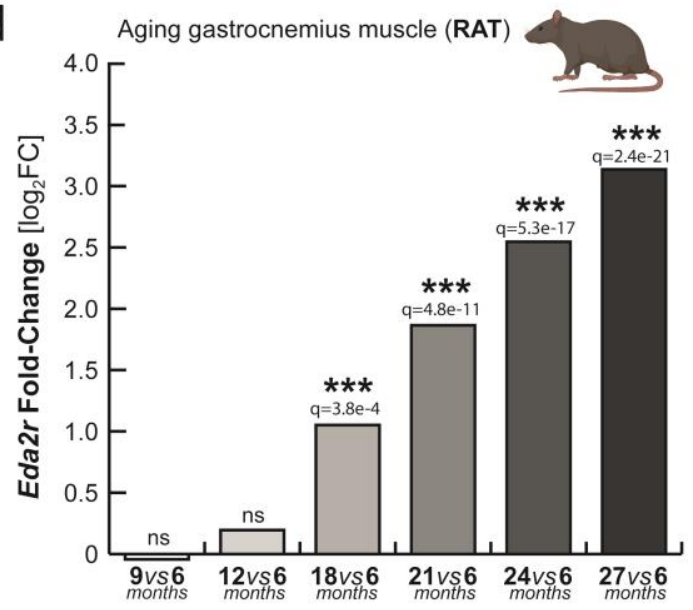

Figure 1

\section{EDA2R remarkably associates with age and is maintained across species.}

125 A) Boxplots representing Pearson's correlation coefficients between mRNA expression of all quantified human genes with sample age, as determined from the GTEx collection of human 127 tissue samples (RNA-Seq). Tissues were evaluated separately as indicated. The yellow dot 128 indicates the relative positioning of $E D A 2 R$ as obtained by ranking genes by their correlation coefficient with donors' age. In several tissues, EDA2R scores first being the most correlated gene, and in most scenarios, its ranking is among the top 10 genes. For ranking positions exceeding the $50^{\text {th }}$, the reference quartile is reported, with Q1 indicating the highest quartile 
132 and Q4 the lowest referred to each tissue. B) Representation of the median Pearson's 133 correlation coefficient for each gene (dots) in all solid tissues. Red dots represent genes 134 increasing with age (positive median correlations), blue represent genes decreasing with age

135 (negative median correlations). Yellow dot indicates EDA2R, which scores as the top ranking 136 gene having high correlation coefficients in all tissues considered. C) Left, RMA normalized 137 expression of Eda2r measured by microarrays (intensities). Boxplots compare murine 138 gastrocnemius samples derived from young and old mice (respectively 10 weeks and 24 139 months; 3 replicates in each group). Adjusted p-value (q-value) is indicated and conventionally 140 represented with ${ }^{* *}$ if minor than 0.01 . Right, The panel shows significance levels ($14110 x \log _{10}$ FDR) for all genes. Eda2r relative positioning is indicated in yellow. D) Changes in 142 Eda2r expression ( $\log _{2}$ fold-change) in rat gastrocnemius comparing older individuals versus 1436 months old rats (ages are reported below each bar). Significance is indicated with FDR 144 adjusted $p$-value ( $q$-value) and represented with *** if minor than 0.001 , not significant (ns) if 145 major than 0.05 . 
a

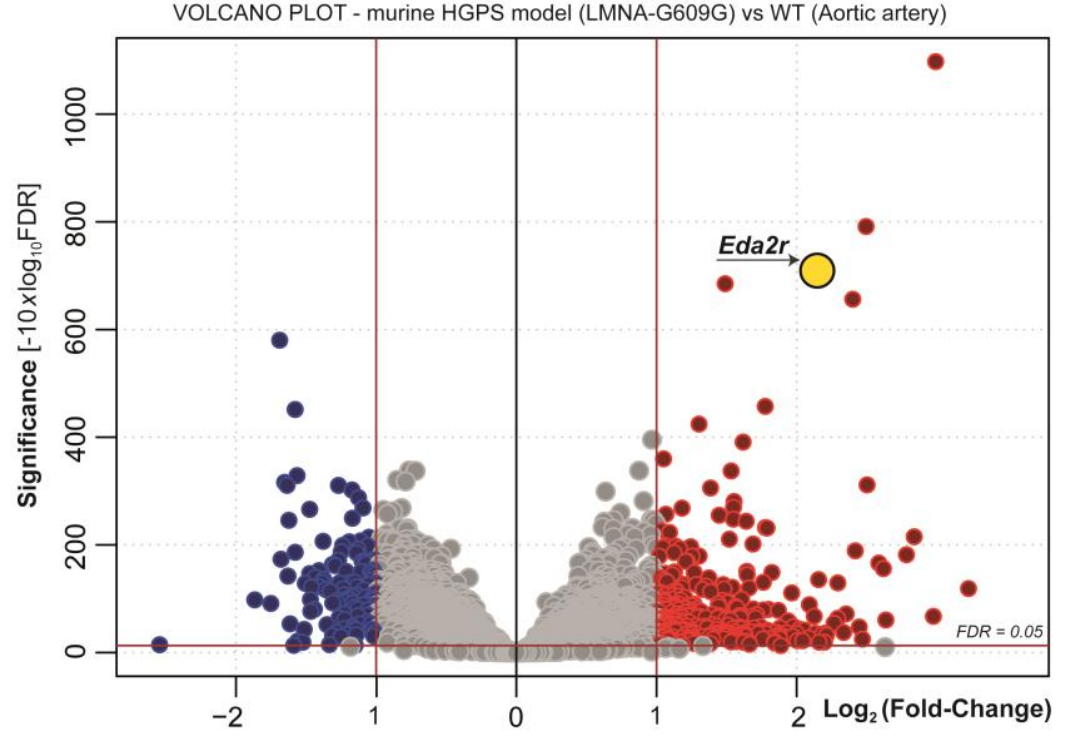

b

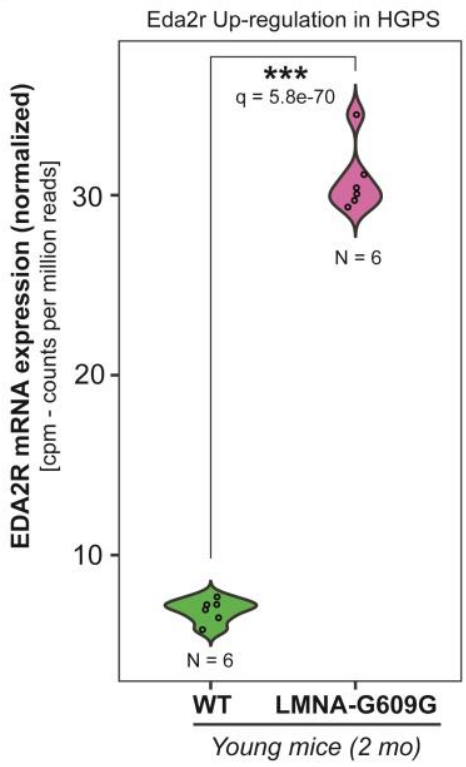

Figure 2

150 Eda2r is among the most upregulated genes in murine models of Hutchinson151 Gilford progeria syndrome (HGPS). A) Volcano plot representing changes in the gene 152 expression of murine aortic artery comparing HGPS, 2 months old mice (LMNA-G609G 153 mutant) against wild type, 2 months old age-matched mice. $\log _{2}$ fold-change and 154 significance $\left(-10 \times \log _{10} \mathrm{FDR}\right)$ are represented. In yellow, as the third most significant 155 differentially expressed gene, Eda2r, which is strongly upregulated in the HGPS group. B) 156 Boxplots representing Eda2r expression in wild type and HGPR mice (LMNA-G609G) (6 157 replicates in each group). Significance is reported with FDR-adjusted p-value (q-value) 158 and conventionally represented with ${ }^{* * *}$ if minor than 0.001 . 
160

\section{Figure 3}

a

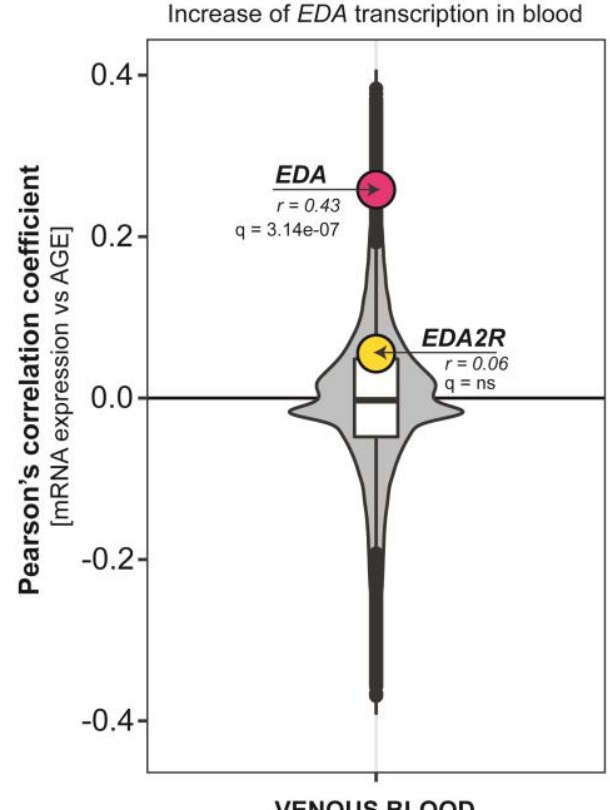

b

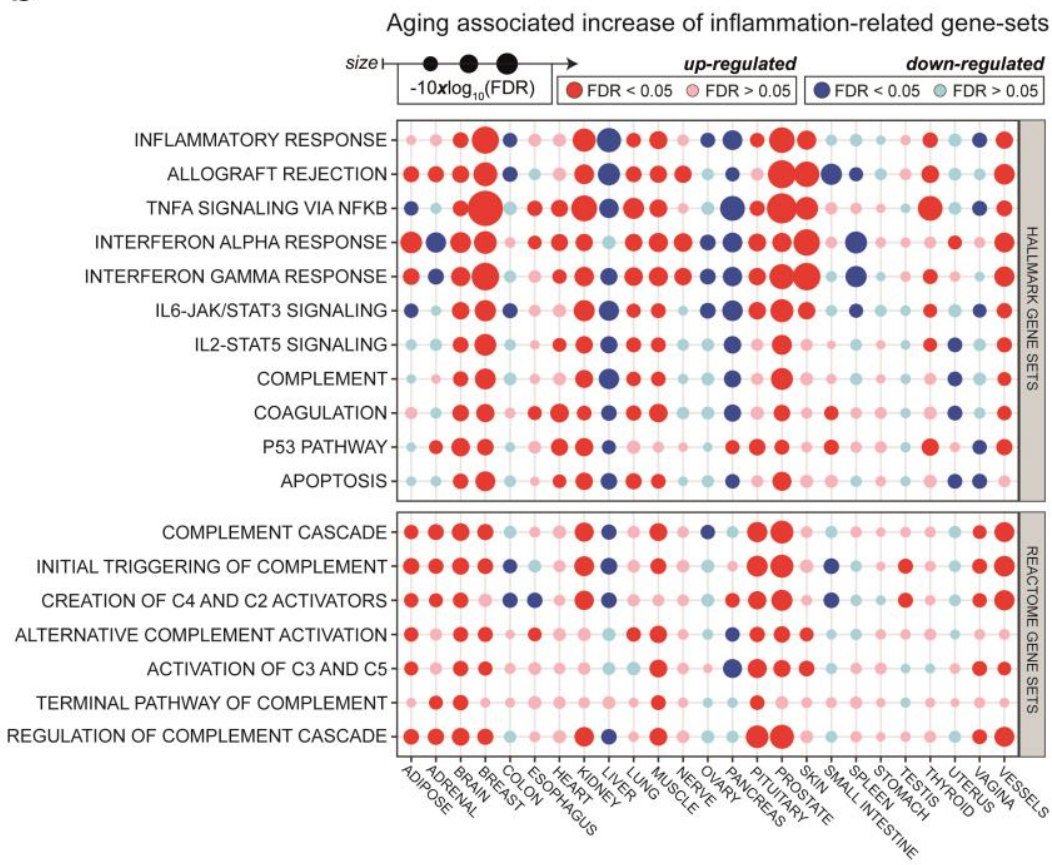

Figure 3
162

163 Age positively correlates with EDA (EDA2R ligand) in human blood and ageing 164

165

166

167

168

169

170

171

172

173

174

175 associates with up-regulation of inflammation-related gene sets in human tissues.

A) Violin plot representing Pearson's correlations between expression and age for all human genes in blood (GTEx dataset). Yellow dot represents EDA2R (ranking within the first quartile), magenta dot indicates the EDA associated correlation coefficient. Coefficients $(r)$ and adjusted $\mathrm{p}$-values (q-values) are reported for both genes. While EDA2R shows less correlation compared with its behavior in solid tissues, EDA is among the most positively correlated genes. B) Aging-dependent perturbations of Hallmark and Reactome inflammation-related gene sets in all human tissues. Circle dimensions are proportional to statistical significance expressed as $-10 x \log _{10} F D R$, colors represent positive (red) and negative (blue) correlations. Gene-sets reaching showing FDR $<0.05$ are depicted with high saturation, not significant genes with low saturation. While inflammatory pathways increase with age in most tissues, a decrease is observed in selective tissues like liver and pancreas. 
References

1 Tanikawa, C., Ri, C., Kumar, V., Nakamura, Y. \& Matsuda, K. Crosstalk of EDAA2/XEDAR in the p53 signaling pathway. Mol Cancer Res 8, 855-863, doi:10.1158/1541-7786.MCR-09-0484 (2010).

2 Brosh, R. et al. p53-dependent transcriptional regulation of EDA2R and its involvement in chemotherapy-induced hair loss. FEBS Lett 584, 2473-2477, doi:10.1016/j.febslet.2010.04.058 (2010).

3 Sinha, S. K., Zachariah, S., Quinones, H. I., Shindo, M. \& Chaudhary, P. M. Role of TRAF3 and -6 in the activation of the NF-kappa B and JNK pathways by Xlinked ectodermal dysplasia receptor. J Biol Chem 277, 44953-44961, doi:10.1074/jbc.M207923200 (2002).

4 Hayden, M. S. \& Ghosh, S. Regulation of NF-kappaB by TNF family cytokines. Semin Immunol 26, 253-266, doi:10.1016/j.smim.2014.05.004 (2014).

5 Song, L. et al. Macrophage-derived EDA-A2 inhibits intestinal stem cells by targeting miR-494/EDA2R/beta-catenin signaling in mice. Commun Biol 4, 213, doi:10.1038/s42003-021-01730-0 (2021).

6 Verhelst, K. et al. XEDAR activates the non-canonical NF-kappaB pathway. Biochem Biophys Res Commun 465, 275-280, doi:10.1016/j.bbrc.2015.08.019 (2015).

7 Kwack, M. H., Kim, J. C. \& Kim, M. K. Ectodysplasin-A2 induces apoptosis in cultured human hair follicle cells and promotes regression of hair follicles in mice. Biochem Biophys Res Commun 520, 428-433, doi:10.1016/j.bbrc.2019.10.031 (2019).

8 Chang, B., Punj, V., Shindo, M. \& Chaudhary, P. M. Adenoviral-mediated gene transfer of ectodysplasin-A2 results in induction of apoptosis and cell-cycle arrest in osteosarcoma cell lines. Cancer Gene Ther 14, 927-933, doi:10.1038/sj.cgt.7701078 (2007).

9 Yang, L., Huang, X., Wang, W., Jiang, T. \& Ding, F. XEDAR inhibits the proliferation and induces apoptosis of gastric cancer cells by regulating JNK signaling pathway. Biosci Rep 39, doi:10.1042/BSR20192726 (2019).

10 Lan, X. et al. EDA2R mediates podocyte injury in high glucose milieu. Biochimie 174, 74-83, doi:10.1016/j.biochi.2020.04.003 (2020).

11 Prodi, D. A. et al. EDA2R is associated with androgenetic alopecia. J Invest Dermatol 128, 2268-2270, doi:10.1038/jid.2008.60 (2008).

12 Richards, J. B. et al. Male-pattern baldness susceptibility locus at 20p11. Nat Genet 40, 1282-1284, doi:10.1038/ng.255 (2008).

13 de Vries, M. et al. Lung tissue gene-expression signature for the ageing lung in COPD. Thorax, doi:10.1136/thoraxjnl-2017-210074 (2017).

14 Carithers, L. J. et al. A Novel Approach to High-Quality Postmortem Tissue Procurement: The GTEx Project. Biopreserv Biobank 13, 311-319, doi:10.1089/bio.2015.0032 (2015).

15 Horvath, S. DNA methylation age of human tissues and cell types. Genome Biol 14, R115, doi:10.1186/gb-2013-14-10-r115 (2013).

16 Hannum, G. et al. Genome-wide methylation profiles reveal quantitative views of human aging rates. Mol Cell 49, 359-367, doi:10.1016/j.molcel.2012.10.016 (2013). 


\section{Methods}

223 Data analysis workflow: RNA-Seq raw counts data were retrieved from GTEx

224 v8 ${ }^{1} .17382$ samples from 980 healthy individuals, derived from the whole variety of tissues

225 included within this transcriptional collection of samples were parsed. Analyses were

226 performed in $\mathrm{R}$ statistical environment and sequencing counts were normalized and

227 further processed using the variable stabilizing transformation (VST) algorithm embedded

228 within the DESeq2 package ${ }^{2}$. We focused at removing batch effects prior to performing

229 tissue-specific correlation to age.

Batch effects corrections and transcriptional correlations to age: Batch effects were

232 explored based on Principal Component analyses (PCA), which were performed

233 separately for every tissue (the top 1000 most variable genes were used for the

234 dimensionality reduction). Being gender and the anatomical sub-localization within the

235 same tissue-type (i.e., Brain region) the most prominent sources of variability, data were

236 corrected for these categorical variables using the removeBatchEffect algorithm, included

237 in the R package limma ${ }^{3}$. Importantly, when adjusting for these features, we defined the

238 biological age of the donor as the batch covariate in the design matrix, making thereby

239 sure that age-related differences would not be biased by batch corrections.

240 Subsequently. for each tissue individually, we computed Pearson's correlations between

241 gene expression and age (represented in years) for all available samples. We derived p-

242 values associated to correlation coefficients and for each tissue considered, we adjusted

243 p-values for multiple testing using the false discovery rate (FDR) method.

\section{Gene-set enrichment analysis}

246 Briefly, for each tissue, we ranked genes based on the adjusted p-value resulting from

247 their correlation with the age of individuals. Gene-set enrichment analysis was performed 
248 using Camera ${ }^{4}$ on Hallmarks (MSigDB) and Reactome gene-sets.

250 Data sources and reprocessing

251 RNA-Seq data for human samples were retrieved from GTEx database and processed as

252 described in the Data analysis workflow section. Gene expression data (Affymetrix Mouse

253 Genome 4302.0 Array) for the gastrocnemius muscle of young (10 weeks) and old (24

254 months) mice were obtained from GSE52550. Data were reprocessed from raw files using

255 the affy package ${ }^{5}$ and normalized using the Robust Multi-Array expression measure using

256 sequence information provided by the gcrma package ${ }^{6}$. Downstream differential

257 expression was performed using limma. RNA-Seq data for aging rat tissues were retrieved

258 from SRA project PRJNA516151. Differential expression results of all genes are provided

259 by the authors and are accessible in from of supplementary files from the respective

260 publication ${ }^{7}$. RNA-Seq data for LMNA-G609G murine models of Hutchinson-Gilford

261 Progeria Syndrome (HGPS) were retrieved from GSE165409. Sequencing reads from raw

262 fastq files were aligned to the GRCm39 release of the reference mouse genome (primary

263 assembly) and annotated using the latest version Gencode GTF file (M27). Reads counts

264 where then processed in R Statistical environment and downstream analysis was

265 performed through the DESeq2 pipeline. 
2711 Carithers, L. J. et al. A Novel Approach to High-Quality Postmortem Tissue 272 Procurement: The GTEx Project. Biopreserv Biobank 13, 311-319, 273 doi:10.1089/bio.2015.0032 (2015).

2742 Love, M. I., Huber, W. \& Anders, S. Moderated estimation of fold change and 275 dispersion for RNA-seq data with DESeq2. Genome Biol 15, 550, 276 doi:10.1186/s13059-014-0550-8 (2014).

2773 Ritchie, M. E. et al. limma powers differential expression analyses for RNA278 sequencing and microarray studies. Nucleic Acids Res 43, e47,

2804 Wu, D. \& Smyth, G. K. Camera: a competitive gene set test accounting for inter281 gene correlation. Nucleic Acids Res 40, e133, doi:10.1093/nar/gks461 (2012). 2825 Gautier, L., Cope, L., Bolstad, B. M. \& Irizarry, R. A. affy--analysis of Affymetrix 283 GeneChip data at the probe level. Bioinformatics 20, 307-315, doi:10.1093/bioinformatics/btg405 (2004).

6 Gharaibeh, R. Z., Fodor, A. A. \& Gibas, C. J. Background correction using dinucleotide affinities improves the performance of GCRMA. BMC Bioinformatics 9, 452, doi:10.1186/1471-2105-9-452 (2008).

7 von Kleeck, R. et al. Arterial stiffness and cardiac dysfunction in HutchinsonGilford Progeria Syndrome corrected by inhibition of lysyl oxidase. Life Sci Alliance 4, doi:10.26508/lsa.202000997 (2021). 


\section{Supplementary Files}

This is a list of supplementary files associated with this preprint. Click to download.

- SupplementaryFigures.pdf

- SuppTable1.xlsx

- SuppTable2.xlsx

- SuppTable3.xlsx 